\title{
Effect of Polymer Coating, Plant Biocides and Insecticide on Seed Quality of Fodder Cowpea during Storage
}

\author{
R. Renuka*, Vinodkumar* and B. S. Vyakaranahal \\ Department of Seed Science and Technology, College of Agriculture, UAS, \\ Dharwad-580005, Karnataka, India \\ *Corresponding author
}

\section{A B S T R A C T}

Keywords

Biocides,

Insecticides,

Polymer coating,

Germination, Cloth

bag, HDPE bag and

Seedling vigour

index

Article Info

Accepted:

05 February 2020

Available Online:

10 March 2020
The laboratory experiment was conducted at the Seed Quality Research Laboratory of National Seed Project, Seed Unit. University of Agricultural Sciences, Dharwad during 2016-2017 to evaluate effect of polymer coating, plant biocides and insecticide on seed quality of fodder cowpea during storage. The experiment consisted of totally 16 treatment combinations involving two factors viz., first factor consists of two containers ( $\mathrm{C}_{1}$ :Cloth bag and $\mathrm{C}_{2}$ :HDPE bag) and second factor consists of 8 seed treatments viz.,[ $\mathrm{T}_{1}$ : Control, $\mathrm{T}_{2}$ : Polymer seed coating@ $5 \mathrm{mlkg}^{-1}$ of seed, $\mathrm{T}_{3}$ : Vitavax(Carboxin 37.5\%@2 $\mathrm{gkg}^{-1}$ of seeds + Thiram@2 $\mathrm{gkg}^{-1}$ of seed), $\mathrm{T}_{4}$ : Aluminium phosphide @ 1 tablet $/ 250 \mathrm{cuft}, \mathrm{T}_{5}$ :Methomyl @ $0.6 \mathrm{gkg}^{-1}$ of seed, $\mathrm{T}_{6}$ : Neem oil @ $5 \mathrm{mlkg}^{-1}$ of seed, $\mathrm{T}_{7}$ : Castor oil @ $5 \mathrm{mlkg}^{-1}$ of seed , $\mathrm{T}_{8}$ : Sweet flag @ $5 \mathrm{gkg}^{-1}$ of seed. Among all the treatments $\mathrm{T}_{3}$ (Vitavax (Carboxin 37.5\%@2 $\mathrm{gkg}^{-1}$ of seeds + Thiram @ $2 \mathrm{gkg}^{-1}$ of seed) showed maximum seed quality parameters like germination $(89.83 \%)$, Root length $(18.76 \mathrm{~cm})$, shoot length $(15.60 \mathrm{~cm})$,seedling vigour index $(3230)$. Where as the lowest germination $(80.00 \%)$, root length $(14.40 \mathrm{~cm})$, shoot length $(11.26 \mathrm{~cm})$, seedling vigour index (2125) were recorded in control at the end of nine months of storage period.

\section{Introduction}

Cowpea (Vigna unguiculata (L.) is one of the most important pulse legume. It is native to central Africa, belongs to the family Fabaceae. Pulses are excellent sources of proteins (20-40\%), carbohydrates (50 - $60 \%)$ and are fairly good sources of thiamin, niacin, calcium and iron. Hence, they form an integral part of our diet and also they occupy major portion of agricultural land and are the second most important group of crops worldwide (Arora, 1989). Apart from its protein content, pulses due to their nitrogen fixing ability, enrich soil fertility. One of the major constraints in storing the cowpea seeds from harvest to next sowing is storage insect pests which inflict severe losses. Pulse beetle is a 
major pest that causes serious damage and is a cosmopolitan. The pulse seed suffer a great damage during storage due to insect attack. Several bruchid species attack cereals and pulses in the store and causes a loss of 10$15 \%$ with a germination loss ranging from 50 92.

Cowpea is no exception because of its high protein content, cowpea seed is highly affected by storage pests and other microflora. The pulse beetle (Callosobruchus chinensis) in storage causes considerable losses and affects the quality of seed. The taking care of seeds during storage, therefore is of supreme importance for India's economic well-being and self-sufficiency.

Information on seed treatment of fodder cowpea with different organic and in-organic source is quite meagre and scanty. Hence, studies on seed storability of fodder cowpea using different organic and inorganic sources of seed treatment was conducted to assess the storability of fodder cowpea seeds using cloth bag and high density polythene bag (HDPE) under ambient conditions of Dharwad.

\section{Materials and Methods}

The experiment was conducted in the Seed Quality Research Laboratory of National Seed Project, Seed unit, University of Agricultural Sciences, Dharwad on influence of polymer coating, plant biocides and insecticide on seed quality of fodder cowpea during storage during 2016 and 2017. Cowpea (Cv.MFC 9-1) Seeds were obtained from Indian Grassland and Fodder Research Institute, Southern Regional Research Station, Dharwad.

The experiment consisted of two factors, factor on is containers viz. Cloth $\operatorname{bag}\left(\mathrm{C}_{1}\right)$ and HDPE bag $\left(\mathrm{C}_{2}\right)$. The second factor deals with seed treatments viz., $\mathrm{T}_{1}$ : Control, $\mathrm{T}_{2}$ : Polymer seed coating@ $9 \mathrm{ml} / \mathrm{kg}$ of seed, $\mathrm{T}_{3}$ : Vitavax
(Carboxin 37.5\%) @ $2 \mathrm{~g} / \mathrm{kg}$ of seeds + Thiram@ @ g/kg of seed), $\mathrm{T}_{4}$ : Aluminium phosphide@1 tablet/250 cuft, T5: Methomyl @ $0.6 \mathrm{~g} / \mathrm{kg}$ of seed, $\mathrm{T}_{6}$ : Neem oil @ $5 \mathrm{ml} / \mathrm{kg}$ of seed, $\mathrm{T}_{7}$ : Castor oil @ $5 \mathrm{ml} / \mathrm{kg}$ of seed, $\mathrm{T}_{8}$ : Sweet flag @ $5 \mathrm{~g} / \mathrm{kg}$ of seed.

Observations on seed quality parameters were recorded monthly. The mean data obtained from the experiment was statistically analysed and subjected to the Analysis of variance by adopting appropriate statistical methods as outlined by Panse and Sukhatme (1967). The critical differences were calculated at one per cent level of significance. The percentage data of germination were transformed into arcsine root transformation before analysis.

\section{Results and Discussion}

\section{Germination (\%)}

The results of germination percentage as influenced by effect of polymer coating, plant biocides and insecticide on seed quality of fodder cowpea during storage are presented in (Table 1). Significant differences in germination percentage due to seed treatments were recorded from fifth month onwards till the end of the storage period.

Significantly highest germination $(94.0 \%)$ was recorded at $5^{\text {th }}$ month in $\mathrm{T}_{3}$ (Carboxin $37.5 \% @ 2 \mathrm{gkg}^{-1}$ of seeds + Thiram @ $2 \mathrm{gkg}^{-}$ ${ }^{1}$ of seeds) which was on par with $\mathrm{T}_{7}$ (Castor oil @ $5 \mathrm{mlkg}^{-1}$ of seed) of 93.3 per cent and $\mathrm{T}_{8}$ (Sweet flag@ $5 \mathrm{gkg}^{-1}$ of seed) 92.8 per cent, while significantly lowest germination per cent was recorded in $\mathrm{T}_{1}$ - control $(89.2 \%)$.

The germination percentage does not differ significantly due to containers. Higher germination was recorded in $\mathrm{C}_{2}$ (HDPE bag) $(84.7 \%)$ and lower germination was recorded in $\mathrm{C}_{1}$ (cloth bag) $(84.0 \%)$ at the end of nine months of storage period. 


\begin{tabular}{|c|c|c|c|c|c|c|c|c|c|c|c|}
\hline \multicolumn{2}{|c|}{ Treatments } & \multicolumn{5}{|c|}{ Germination (\%) } & \multicolumn{5}{|c|}{ Root length $(\mathrm{cm})$} \\
\hline & & \multicolumn{5}{|c|}{ Months after storage } & \multicolumn{5}{|c|}{ Months after storage } \\
\hline & & Initial month & $3^{\text {rd }}$ month & $5^{\text {th }}$ month & $7^{\text {th }}$ month & $9^{\text {th }}$ month & $\begin{array}{l}\text { Initial } \\
\text { month }\end{array}$ & $3^{\text {rd }}$ month & $5^{\text {th }}$ month & $7^{\text {th }}$ month & $9^{\text {th }}$ month \\
\hline \multirow{5}{*}{$\begin{array}{l}\text { Containers } \\
\text { (C) }\end{array}$} & $\left(\mathrm{C}_{1}\right)$ & $95.6(78.0)$ & $94.3(76.3)$ & $91.1(72.8)$ & $88.3(70.5)$ & $84.0(66.0)$ & 21.00 & 19.47 & 18.42 & 16.81 & 15.44 \\
\hline & $\left(\mathrm{C}_{2}\right)$ & $95.9(78.2)$ & $94.7(76.8)$ & $92.0(73.6)$ & $88.8(70.6)$ & $84.7(67.1)$ & 21.05 & 19.49 & 18.57 & 16.96 & 15.66 \\
\hline & Mean & $95.8(78.2)$ & $94.5(76.5)$ & $91.5(73.2)$ & $88.6(70.3)$ & $84.3(66.8)$ & 21.02 & 19.48 & 18.49 & 16.89 & 15.55 \\
\hline & SEm \pm & 0.24 & 0.35 & 0.28 & 0.14 & 0.21 & 0.14 & 0.14 & 0.07 & 0.06 & 0.09 \\
\hline & $\mathrm{CD}(0.01)$ & NS & NS & NS & NS & NS & NS & NS & NS & NS & NS \\
\hline \multirow{10}{*}{$\begin{array}{l}\text { Treatments } \\
\text { (T) }\end{array}$} & $\mathrm{T}_{1}$ & $94.8(76.9)$ & $93.3(75.4)$ & $89.2(71.0)$ & $85.3(67.5)$ & $80.0(63.5)$ & 20.86 & 19.16 & 17.22 & 15.98 & 14.04 \\
\hline & $\mathrm{T}_{2}$ & $95.7(78.0)$ & $94.3(76.2)$ & $91.0(72.6)$ & $86.5(68.4)$ & $82.2(65.0)$ & 21.03 & 19.51 & 18.62 & 16.73 & 15.29 \\
\hline & $\mathrm{T}_{3}$ & $96.7(79.5)$ & $96.0(78.6)$ & $94.0(75.8)$ & $92.0(73.6)$ & $89.8(71.4)$ & 21.09 & 19.58 & 18.76 & 17.34 & 16.19 \\
\hline & $\mathrm{T}_{4}$ & $95.7(78.0)$ & $94.0(75.8)$ & $89.5(71.1)$ & $86.0(68.0)$ & $81.0(64.2)$ & 21.02 & 19.50 & 18.61 & 16.65 & 15.24 \\
\hline & $\mathrm{T}_{5}$ & $95.2(77.3)$ & $93.8(75.6)$ & $89.8(71.4)$ & $85.8(67.9)$ & $80.7(63.9)$ & 21.01 & 19.48 & 18.60 & 16.63 & 15.18 \\
\hline & $\mathrm{T}_{6}$ & $95.8(78.3)$ & $94.5(76.4)$ & $92.5(74.1)$ & $90.5(72.0)$ & $86.3(68.3)$ & 21.05 & 19.53 & 18.69 & 17.23 & 16.13 \\
\hline & $\mathrm{T}_{7}$ & $96.2(78.7)$ & $95.3(77.5)$ & $93.3(75.0)$ & $91.3(72.9)$ & $88.0(69.7)$ & 21.08 & 19.56 & 18.73 & 17.29 & 16.18 \\
\hline & $\mathrm{T}_{8}$ & $96.2(78.7)$ & $94.8(76.9)$ & $92.8(74.5)$ & $91.0(72.5)$ & $86.8(68.7)$ & 21.07 & 19.54 & 18.71 & 17.25 & 16.15 \\
\hline & SE $m \pm$ & 0.48 & 0.70 & 0.56 & 0.28 & 0.43 & 0.05 & 0.13 & 0.14 & 0.11 & 0.18 \\
\hline & $\mathrm{CD}(0.01)$ & NS & NS & 2.17 & 1.08 & 1.65 & NS & NS & 0.54 & 0.43 & 0.70 \\
\hline \multirow{3}{*}{$\begin{array}{l}\text { Interaction } \\
(\mathrm{CX} \mathrm{T})\end{array}$} & $\mathbf{C}_{1} \mathbf{T}_{1}$ & 94.7 (76.7) & $93.3(75.7)$ & $89.0(71.0)$ & $85.3(67.5)$ & $80.0(63.5)$ & 20.83 & 19.13 & 16.70 & 15.45 & 13.22 \\
\hline & $\mathbf{C}_{1} \mathbf{T}_{2}$ & 95.7 (78.0) & $94.0(75.8)$ & $90.0(71.6)$ & $86.3(68.3)$ & $82.0(65.0)$ & 21.01 & 19.50 & 18.60 & 16.72 & 15.28 \\
\hline & $\mathrm{C}_{1} \mathrm{~T}_{3}$ & $96.33(79.0)$ & $95.67(78.0)$ & $93.67(75.4)$ & $91.67(73.2)$ & 89.67 (71.2) & 21.06 & 19.57 & 18.76 & 17.33 & 16.19 \\
\hline
\end{tabular}




\begin{tabular}{|c|c|c|c|c|c|c|c|c|c|c|}
\hline $\mathrm{C}_{1} \mathrm{~T}_{4}$ & $95.67(78.0)$ & $93.67(75.4)$ & $89.00(70.6)$ & $85.67(67.8)$ & 80.67 (63.9) & 21.00 & 19.48 & 18.58 & 16.63 & 15.21 \\
\hline $\mathrm{C}_{1} \mathrm{~T}_{5}$ & $95.00(77.1)$ & 93.67 (75.4) & $89.67(71.2)$ & $85.67(67.8)$ & $80.33(63.7)$ & 21.00 & 19.47 & 18.57 & 16.62 & 15.16 \\
\hline $\mathrm{C}_{1} \mathrm{~T}_{6}$ & $95.67(78.0)$ & $94.33(76.2)$ & $92.00(73.6)$ & $90.33(71.9)$ & $85.67(67.8)$ & 21.02 & 19.52 & 18.68 & 17.22 & 16.13 \\
\hline $\mathrm{C}_{1} \mathrm{~T}_{7}$ & $96.00(78.5)$ & $95.00(77.1)$ & $93.00(74.7)$ & $91.00(72.5)$ & 87.67 (69.4) & 21.05 & 19.55 & 18.73 & 17.28 & 16.17 \\
\hline $\mathrm{C}_{1} \mathrm{~T}_{8}$ & $96.00(78.5)$ & $94.67(76.7)$ & $92.33(73.9)$ & $90.67(72.2)$ & $86.33(68.3)$ & 21.05 & 19.53 & 18.71 & 17.24 & 16.15 \\
\hline $\mathrm{C}_{2} \mathrm{~T}_{1}$ & $95.00(77.2)$ & $93.33(75.0)$ & $89.33(70.9)$ & $85.33(67.5)$ & $80.00(63.4)$ & 20.89 & 19.20 & 17.74 & 16.51 & 14.86 \\
\hline $\mathrm{C}_{2} \mathrm{~T}_{2}$ & $95.67(78.0)$ & 94.67 (76.7) & $92.00(73.6)$ & $86.67(68.6)$ & $82.33(65.1)$ & 21.05 & 19.52 & 18.63 & 16.74 & 15.30 \\
\hline $\mathrm{C}_{2} \mathrm{~T}_{3}$ & $97.00(80.1)$ & $96.33(79.2)$ & $94.33(76.2)$ & $92.33(73.9)$ & $90.00(71.6)$ & 21.11 & 19.58 & 18.77 & 17.35 & 16.20 \\
\hline $\mathrm{C}_{2} \mathrm{~T}_{4}$ & $95.67(78.0)$ & $94.33(76.2)$ & $90.00(71.6)$ & $86.33(68.3)$ & $81.33(64.4)$ & 21.03 & 19.51 & 18.63 & 16.66 & 15.26 \\
\hline $\mathrm{C}_{2} \mathrm{~T}_{5}$ & $95.33(77.5)$ & $94.00(75.8)$ & $90.00(71.6)$ & $86.00(68.0)$ & $81.00(64.2)$ & 21.01 & 19.49 & 18.62 & 16.64 & 15.20 \\
\hline $\mathrm{C}_{2} \mathrm{~T}_{6}$ & $96.00(78.5)$ & 94.67 (76.7) & $93.00(74.7)$ & $90.67(72.2)$ & $87.00(68.9)$ & 21.07 & 19.53 & 18.70 & 17.23 & 16.14 \\
\hline $\mathrm{C}_{2} \mathrm{~T}_{7}$ & $96.33(79.0)$ & $95.67(78.0)$ & $93.67(75.4)$ & $91.67(73.2)$ & $88.33(70.0)$ & 21.10 & 19.56 & 18.74 & 17.30 & 16.18 \\
\hline $\mathrm{C}_{2} \mathrm{~T}_{8}$ & $96.33(79.0)$ & $95.00(77.1)$ & $93.33(75.0)$ & $91.33(72.9)$ & $87.33(69.1)$ & 21.09 & 19.54 & 18.72 & 17.26 & 16.15 \\
\hline Mean & 95.77 (78.2) & $94.52(76.5)$ & 91.52 (73.2) & $88.56(70.3)$ & $84.35(66.8)$ & 21.02 & 19.48 & 18.49 & 16.89 & 15.55 \\
\hline SE $m \pm$ & 0.67 & 0.99 & 0.79 & 0.39 & 0.60 & 0.06 & 0.18 & 0.20 & 0.16 & 0.26 \\
\hline $\mathrm{CD}(0.01)$ & NS & NS & NS & NS & NS & NS & NS & NS & NS & NS \\
\hline
\end{tabular}




\begin{tabular}{|c|c|c|c|c|c|c|c|c|c|c|c|}
\hline \multirow{3}{*}{\multicolumn{2}{|c|}{ Treatments }} & \multirow{2}{*}{\multicolumn{5}{|c|}{$\begin{array}{l}\text { Shoot length }(\mathrm{cm}) \\
\text { Months after storage }\end{array}$}} & \multirow{2}{*}{\multicolumn{5}{|c|}{$\begin{array}{l}\text { Seedling Vigour Index } \\
\text { Months after storage }\end{array}$}} \\
\hline & & & & & & & & & & & \\
\hline & & $\begin{array}{l}\text { Initial } \\
\text { month }\end{array}$ & $\begin{array}{l}3^{\text {rd }} \\
\text { month }\end{array}$ & $\begin{array}{c}5^{\text {th }} \\
\text { month }\end{array}$ & $\begin{array}{l}7^{\text {th }} \\
\text { month }\end{array}$ & $\begin{array}{l}9^{\text {th }} \\
\text { month }\end{array}$ & $\begin{array}{l}\text { Initial } \\
\text { month }\end{array}$ & $\begin{array}{c}3^{\text {rd }} \\
\text { month }\end{array}$ & $\begin{array}{c}5^{\text {th }} \\
\text { month }\end{array}$ & $\begin{array}{l}7^{\text {th }} \\
\text { month }\end{array}$ & $\begin{array}{c}9^{\text {th }} \\
\text { month }\end{array}$ \\
\hline \multirow{5}{*}{$\begin{array}{l}\text { Containers } \\
\text { (C) }\end{array}$} & $\left(\mathrm{C}_{1}\right)$ & 16.50 & 16.06 & 15.07 & 14.08 & 12.48 & 3586 & 3349 & 3072 & 2731 & 2399 \\
\hline & $\left(\mathrm{C}_{2}\right)$ & 16.55 & 16.11 & 15.18 & 14.19 & 12.56 & 3607 & 3373 & 3130 & 2768 & 2451 \\
\hline & Mean & 16.53 & 16.08 & 15.12 & 14.13 & 12.52 & 3596 & 3361 & 3101 & 2750 & 2425 \\
\hline & SE m \pm & 0.05 & 0.03 & 0.06 & 0.06 & 0.07 & 26 & 22 & 20 & 18 & 16 \\
\hline & $\mathrm{CD}(0.01)$ & NS & NS & NS & NS & NS & NS & NS & NS & NS & NS \\
\hline \multirow{10}{*}{$\begin{array}{l}\text { Treatments } \\
\text { (T) }\end{array}$} & $\mathrm{T}_{1}$ & 16.33 & 15.87 & 13.97 & 12.56 & 11.26 & 3527 & 3265 & 2796 & 2436 & 2125 \\
\hline & $\mathrm{T}_{2}$ & 16.48 & 16.08 & 14.95 & 14.02 & 12.36 & 3588 & 3358 & 3093 & 2660 & 2373 \\
\hline & $\mathrm{T}_{3}$ & 16.83 & 16.20 & 15.60 & 14.67 & 13.01 & 3665 & 3434 & 3230 & 2945 & 2623 \\
\hline & $\mathrm{T}_{4}$ & 16.45 & 16.06 & 14.94 & 14.01 & 12.35 & 3584 & 3342 & 3075 & 2636 & 2345 \\
\hline & $\mathrm{T}_{5}$ & 16.43 & 16.04 & 14.89 & 13.96 & 12.30 & 3563 & 3333 & 3064 & 2625 & 2331 \\
\hline & $\mathrm{T}_{6}$ & 16.50 & 16.11 & 15.52 & 14.59 & 12.93 & 3598 & 3367 & 3165 & 2880 & 2509 \\
\hline & $\mathrm{T}_{7}$ & 16.69 & 16.17 & 15.57 & 14.64 & 12.98 & 3632 & 3406 & 3202 & 2916 & 2566 \\
\hline & $\mathrm{T}_{8}$ & 16.51 & 16.15 & 15.55 & 14.62 & 12.96 & 3614 & 3384 & 3181 & 2900 & 2528 \\
\hline & $\mathrm{SE} \mathrm{m} \pm$ & 0.09 & 0.07 & 0.12 & 0.12 & 0.13 & 52 & 43 & 40 & 35 & 32 \\
\hline & $\mathrm{CD}(0.01)$ & NS & NS & 0.48 & 0.46 & 0.51 & NS & NS & 157 & 136 & 125 \\
\hline \multirow{19}{*}{$\begin{array}{l}\text { Interaction } \\
(\mathrm{CX} T)\end{array}$} & $\mathrm{C}_{1} \mathrm{~T}_{1}$ & 16.20 & 15.75 & 13.03 & 12.19 & 11.02 & 3505 & 3245 & 2704 & 2358 & 2026 \\
\hline & $C_{1} T_{2}$ & 16.47 & 16.07 & 14.45 & 14.00 & 12.34 & 3586 & 3343 & 3073 & 2652 & 2356 \\
\hline & $\mathrm{C}_{1} \mathrm{~T}_{3}$ & 16.82 & 16.19 & 15.12 & 14.67 & 13.01 & 3649 & 3421 & 3218 & 2933 & 2618 \\
\hline & $\mathrm{C}_{1} \mathrm{~T}_{4}$ & 16.44 & 16.04 & 14.44 & 13.99 & 12.33 & 3581 & 3328 & 3048 & 2623 & 2323 \\
\hline & $\mathrm{C}_{1} \mathrm{~T}_{5}$ & 16.42 & 16.03 & 14.39 & 13.94 & 12.28 & 3555 & 3325 & 3032 & 2618 & 2314 \\
\hline & $\mathrm{C}_{1} \mathrm{~T}_{6}$ & 16.49 & 16.10 & 15.03 & 14.58 & 12.92 & 3588 & 3360 & 3146 & 2873 & 2489 \\
\hline & $\mathrm{C}_{1} \mathrm{~T}_{7}$ & 16.68 & 16.17 & 15.09 & 14.64 & 12.98 & 3622 & 3393 & 3189 & 2904 & 2555 \\
\hline & $\mathrm{C}_{1} \mathrm{~T}_{8}$ & 16.50 & 16.14 & 15.06 & 14.61 & 12.95 & 3605 & 3377 & 3163 & 2888 & 2513 \\
\hline & $\mathrm{C}_{2} \mathrm{~T}_{1}$ & 16.46 & 15.98 & 13.72 & 12.94 & 11.51 & 3549 & 3284 & 2887 & 2513 & 2223 \\
\hline & $\mathrm{C}_{2} \mathrm{~T}_{2}$ & 16.48 & 16.09 & 14.49 & 14.04 & 12.38 & 3590 & 3372 & 3113 & 2667 & 2390 \\
\hline & $\mathrm{C}_{2} \mathrm{~T}_{3}$ & 16.84 & 16.21 & 15.13 & 14.68 & 13.02 & 3682 & 3448 & 3243 & 2957 & 2629 \\
\hline & $\mathrm{C}_{2} \mathrm{~T}_{4}$ & 16.46 & 16.07 & 14.48 & 14.03 & 12.37 & 3587 & 3357 & 3102 & 2650 & 2367 \\
\hline & $\mathrm{C}_{2} \mathrm{~T}_{5}$ & 16.45 & 16.05 & 14.43 & 13.98 & 12.32 & 3571 & 3341 & 3096 & 2633 & 2348 \\
\hline & $\mathrm{C}_{2} \mathrm{~T}_{6}$ & 16.51 & 16.11 & 15.05 & 14.60 & 12.94 & 3608 & 3375 & 3184 & 2886 & 2530 \\
\hline & $\mathrm{C}_{2} \mathrm{~T}_{7}$ & 16.70 & 16.18 & 15.10 & 14.65 & 12.99 & 3642 & 3419 & 3214 & 2928 & 2577 \\
\hline & $\mathrm{C}_{2} \mathrm{~T}_{8}$ & 16.52 & 16.15 & 15.08 & 14.63 & 12.97 & 3624 & 3391 & 3199 & 2912 & 2543 \\
\hline & Mean & 16.53 & 16.08 & 15.12 & 14.13 & 12.52 & 3596 & 3361 & 3101 & 2750 & 2425 \\
\hline & SE m \pm & 0.13 & 0.09 & 0.18 & 0.17 & 0.19 & 74 & 61 & 57 & 50 & 46 \\
\hline & $\mathrm{CD}(0.01)$ & NS & NS & NS & NS & NS & NS & NS & NS & NS & NS \\
\hline
\end{tabular}


Interaction effects due to containers and seed treatments recorded non-significant difference throughout the storage period however, the seed treatment $\mathrm{C}_{2} \mathrm{~T}_{3}$ recorded higher germination percentage of 90.0 , which was on par with $\mathrm{C}_{1} \mathrm{~T}_{3}(89.7 \%)$ and $\mathrm{C}_{2} \mathrm{~T}_{8}(88.3 \%)$ and the lowest germination was recorded in $\mathrm{C}_{1} \mathrm{~T}_{1}$ $(80.0 \%)$ at the end of nine month of storage period.

Vitavax power minimizes the impact of ageing enzymes; it also acts as a protective agent against seed deterioration due to fungal invasion and physiological ageing as a result of which the seed viability was maintained comparatively for longer period of time by acting as anti-ageing agent and there by maintained the germination percentage throughout the storage period. These results are in agreement with the findings Sushma (2003) in chickpea; Vinod Kumar et al., (2012) in pigeon pea.

\section{Root length, shoot length and Seedling vigour index}

Significant differences due to seed treatments on root length shoot length and vigour index recorded. Significantly highest root length, shoot length and vigour index was observed $18.76 \mathrm{~cm}, 15.60 \mathrm{~cm}$ and 3230 , respectively was recorded at $5^{\text {th }}$ month in $\mathrm{T}_{3}$ (Carboxin $37.5 \%$ @ $2 \mathrm{gkg}^{-1}$ of seeds + Thiram @ $2 \mathrm{gkg}^{-1}$ of seeds). While the lowest root length (14.04 $\mathrm{cm})$, shoot length $(11.26 \mathrm{~cm})$ and vigour index (2936) was recorded in $\mathrm{T}_{8}$ control (Without treatment).

The root length shoot length and vigour index do not differed significantly due to containers during storage period. At the end of nine months of storage period numerically higher root length $(15.66 \mathrm{~cm})$, shoot length $(12.56$ $\mathrm{cm})$ and vigour index (2451) was recorded in $\mathrm{C}_{2}$ (HDPE bag).
Interaction effects due to containers and seed treatments recorded non-significant difference throughout the storage period however the treatment combination of $\mathrm{C}_{2} \mathrm{~T}_{3}$ recorded higher root length, shoot length and vigour index of $16.20 \mathrm{~cm} 13.02 \mathrm{~cm}$ and 2629, respectively and lowest root length, shoot length and vigour index was recorded in $\mathrm{C}_{1} \mathrm{~T}_{1}$ $13.22 \mathrm{~cm}, 11.02 \mathrm{~cm}$ and 2026, respectively at the end of nine month of storage period.

Different seed treatments showed significant variations in seed qualitative and quantitative parameters throughout the nine months of storage period. The germination percentage $(\%)$, root length $(\mathrm{cm})$, shoot length $(\mathrm{cm})$ and seedling vigour index of cowpea seeds decreased gradually with increase in the period of storage in all the treatment combinations, which might be attributed to the phenomenon of physiological deterioration, natural ageing and depletion of food reserves and might be due to damage caused by fungi and insects and also toxic metabolites which might have hindered the seedling growth, gradual decline in seed vigour index was noticed due to age induced decline in germination, decrease in dry matter accumulation in seedling and decrease in seedling length at the end of nine months of storage. Similar findings are in conformity with findings of Vinodkumar et al., (2010) in cowpea and Omvati and Verma (2014) in soybean.

Based on the above discussion it is clear that from the results obtained, is concluded that irrespective of seed treatments seed quality parameters decreased with the advancement of storage period. Among all the seed treatments, $\mathrm{T}_{3}$ (Carboxin 37.5\% @ of seeds + Thiram @ $2 \mathrm{gkg}^{-1}$ of seeds) gave significantly highest germination percentage root length, shoot length and seedling vigour index at the end of nine month of storage period and 
which was on par withT 7 (Castor oil @ 5 $\mathrm{mlkg}^{-1}$ of seed). While significantly lowest germination percentage was recorded in $T_{1}$ control (Without treatment). However, the minimum germination of $75 \%$ as per the IMSCS (Indian Minimum Seed Certification Standard) was maintained in vitavax power even after nine months of storage period.

\section{References}

Abdul-Baki, A. A. and Anderson, J. D., 1973, Vigour determination in soybean by multiple criteria. Crop Sci., 13: 630637.

Anonymous, 2011, International Rules for Seed Testing. Seed Sci. and Tech., 21: $1-255$.

Arora, P. P., 1989, Genetic divergence studies and scope for improvement in chickpea National Symposium on Few Front line in Pulse Research.

Omvati Verma and Verma R. S., 2014, Effect of seed coating material and storage containers on germination and seedling vigour of soybean (Glycine max 1.). Saarc J. Agri., 12 (2): 16-24.

Panse, V. G. and Sukhatme, P. V., 1967, Statistical Methods for Agricultural Workers, ICAR, New Delhi, 167-174.
Singh, V. N., and Singh, Y. P., 1995, Relative to resistance of gram varieties to Callosobruchus chinensis on the basis of biochemical parameters. Indian J. Entomol., 57: 77-82.

Sushma, D. M., 2003, Effect of seed treatment and containers on seed storability of garden pea (Pisum sativum L.). M. Sc. (Agri.) Thesis, Univ. Agric. Sci., Dharwad.

Upadhayay, R. G., Sharma, S. and Drawal, N. S., 1999, Effect of Rhizobium inoculation and graded level of $\mathrm{P}$ on the growth and yield of green gram, Legume Res., 22: 277-279.

Vinodkumar, S. B., 2012, Effect of plant products, chemicals and polymer coat on seed storability and field performance of pigeon pea [Cajanus cajan (L.) Mill sp.] cv. bsmr-736. $M$. Sc. (Agri.) Thesis, Univ. Agric. Sci., Dharwad.

Vinod Kumar, Sridhar, K., Karthigeyan, S., Kulakarni, N., 2010, Assessing the seed viability of fodder cowpea varieties during storage using plant biocides, fungicides and insecticides. Range management and agroforestry, 1 (1): 41-43.

\section{How to cite this article:}

Renuka. R, Vinodkumar and Vyakaranahal. B. S. 2020. Effect of Polymer Coating, Plant Biocides and Insecticide on Seed Quality of Fodder Cowpea during Storage. Int.J.Curr.Microbiol.App.Sci. 9(03): 962-968. doi: https://doi.org/10.20546/ijcmas.2020.903.113 